\title{
Educational program to suppress the progression of diabetic nephropathy
}

Shota Moyama ${ }^{1,2}$, Naomi Kitatani ${ }^{1}$, Mayuka Sakaguchi ${ }^{1,2}$, Ayumi Okimoto ${ }^{3}$, Miyoko Nishimoto ${ }^{3}$, Noriko Kato ${ }^{3}$, Yoko Yasui $^{3}$, Saki Okamoto $^{2,4}$, Nagaaki Tanaka ${ }^{2,4}$, Takanori Hyo ${ }^{4}$, Takeshi Kurose ${ }^{2,4}$, Yoshiyuki Hamamoto ${ }^{2,4}$, Hitoshi Kuwata ${ }^{1,2,4}$, Yutaka Seino $^{2,4}$

${ }^{1}$ Kansai Electric Power Hospital - Center for Metabolism and Clinical Nutrition, ${ }^{2}$ Kansai Electric Power Medical Research Institute - Yutaka Seino Distinguished Center for Diabetes Research, ${ }^{3}$ Kansai Electric Power Hospital - Department of Nursing, ${ }^{4}$ Kansai Electric Power Hospital - Center for Diabetes, Endocrinology and Metabolism.

Background and Aims: The number of patients with diabetic nephropathy that is a major cause disease of introduction of dialysis is increasing year by year. It is one of the big issue in Japan. Since 2012, multidisciplinary team consisting of physicians, nurses and dietitians has implemented the educational program in our hospital. We aimed to examine the efficacy of our educational program to prevent the progression of diabetic nephropathy.

Methods: [Study design] retrospective study, [Subjects] 100 patients (66 males, 34 females)

Inclusion criteria: early stage of diabetic nephropathy those who had microalbuminuria (eGFR $>30 \mathrm{ml} / \mathrm{min} / 1.73 \mathrm{~m}^{2}$, urinary albumin $30-299 \mathrm{mg} / \mathrm{gCRE}$ ), and who had been started the intervention program during the period of 2012-2014.

[Intervention protocol]

Physicians, nurses, and dietitians worked together on the same day once every 2-3 months.

physicians

checked medical conditions, adjusted medication and

advised lifestyle modifications nurses

confirmed the adherence to the medication, the psychological burden on the treatment, and the implementation of foot care dietitians

evaluated the dietary intakes and advised the dietary balance correction and sodium restriction.
Table 1. Baseline Characteristics of study subjects

\begin{tabular}{lc}
\hline $\mathrm{n}$ (male/female) & $100(66 / 34)$ \\
Age (years) & $67.9 \pm 10.9$ \\
$\mathrm{BMI}\left(\mathrm{kg} / \mathrm{m}^{2}\right)$ & $25.8 \pm 3.7$ \\
$\mathrm{SBP}(\mathrm{mmHg})$ & $130.5 \pm 11.6$ \\
$\mathrm{dBP}(\mathrm{mmHg})$ & $76.0 \pm 8.3$ \\
$\mathrm{HbA} 1 \mathrm{c}(\%)$ & $7.4 \pm 1.0$ \\
$\mathrm{Cre}(\mathrm{mg} / \mathrm{dL})$ & $0.8 \pm 0.2$ \\
$\mathrm{eGFR}\left(\mathrm{ml} / \mathrm{min} / 1.73 \mathrm{~m}^{2}\right)$ & $68.0 \pm 17.8$ \\
$\mathrm{U}-\mathrm{Alb}(\mathrm{mg} / \mathrm{gCRE})$ & $99.3 \pm 89.9$ \\
\hline \multicolumn{2}{c}{} \\
\end{tabular}

In order to verify the effectiveness of this intervention program, we collected HbA1c and eGFR values for 7 years from electronic medical records, and investigated the change rates before 3 years and after 3 years of the interventions.

\section{Results:}

口 Although $\mathrm{HbA1c}$ tended to increase gradually during 7 years (7.4 to $7.6 \%)$, no statistically significant changes were observed (Fig.1).

D eGFR showed a significant decline over the 3 years before the intervention. However, in the 4 years after the intervention, the eGFR decline over time was suppressed (Fig. 2).

口 Compare to the eGFR reduction rates for 3 years before the intervention, the reduction rate of eGFR after the intervention was significantly lower (Fig. 3).

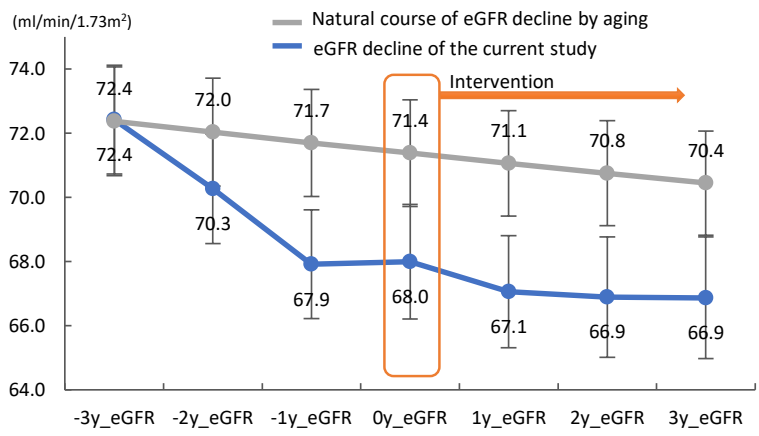

Fig 2. Changes of eGFR compared with natural course.

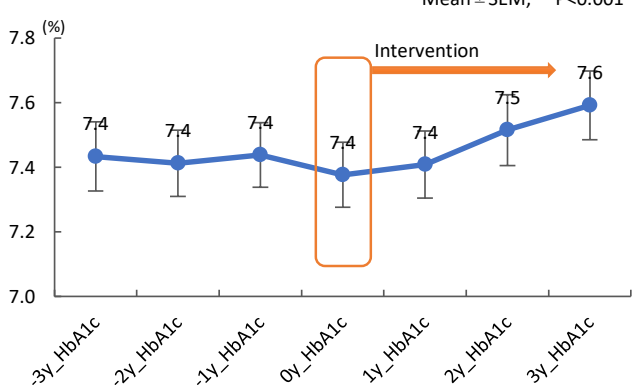

Fig 1. Changes of $\mathrm{HbA} 1 \mathrm{c}$

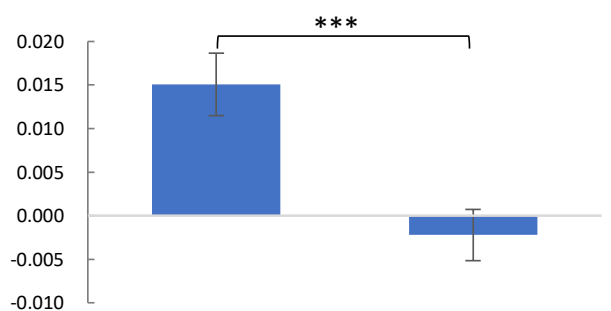

pre-Intervention

post-Intervention

Fig 3. Comparison of eGFR reduction rate

\section{Conclusion:}

It is suggested that a multidisciplinary approach with physicians, nurses and dietitians is effective for preventing the development of early-stage diabetic nephropathy. 